\title{
Ship Electric Propulsion Simulation System Reliability Evaluation Based on Improved D-S Expert Weight Calculation Method
}

\author{
Bing Li, Guoliang Gu, Bowen Xing, and Lihong Li \\ College of Automation, Harbin Engineering University, Harbin, Heilongjiang 150001, China \\ Correspondence should be addressed to Bowen Xing; bwheu@outlook.com
}

Received 12 August 2014; Revised 21 September 2014; Accepted 5 October 2014

Academic Editor: Shaofan Li

Copyright (C) 2015 Bing Li et al. This is an open access article distributed under the Creative Commons Attribution License, which permits unrestricted use, distribution, and reproduction in any medium, provided the original work is properly cited.

\begin{abstract}
In order to have a better evaluation process to determine the experts weight in the evaluation process, this paper proposes a new expert weight calculation method. First of all to establish electric propulsion simulation evaluation system, use AHP method to calculate the initial weight principle of index. Then use the D-S to fuse the experts evaluation information, combined with the weight vector, structure of the expert weight objective function, and through the genetic algorithm to solve the expert weight size. According to the expert weight vector, calculate the final weight vector. Not only can it greatly make use of the experts information and analyze the similarity of information effectively but also it calculates the weight of each expert objectively. At the same time the evaluation subjective factors have been reduced by the adoption of this new method.
\end{abstract}

\section{Introduction}

Regarding ship electric propulsion system as a modern ship career development direction, its safety and reliability are more and more concerned about [1]. The simulation technology is one of the important means for people to study ship electric propulsion system [2]. But the results of simulation credibility are worth considering, and incorrect results could lead to major events. So analyzing the credibility in the system simulation results, determining the relative weight of each subsystem, has great significance for studying the mechanism of ship electric propulsion system.

In the system simulation credibility analysis, we need to make sure of the mutual importance of the systems, namely, the weight. Analytic hierarchy process (AHP) is a comprehensive evaluation method used in research of complicated system [3]. The basic idea of using analytic hierarchy process to determine weight is to invite more related experts to compare each subsystem and to identify and analyse the judgment matrix. As a result of difference between each expert in knowledge, experience, ability, and level, different experts have different result to weight evaluation system. How to make better use of the evaluation experts has always been about the topic of comparison.

The D-S evidence theory is a method widely used in information fusion technology [4]. Chen et al. propose making use of the Markov random fields (MRFs) and D$S$ evidence theory to interactive color image segmentation method [5]. Si et al. proposed a novel prediction approach through information fusion of improved D-S evidence theory and neural network to forecast the distribution of coal seam terrain [6]. Li and Pang use D-S evidence theory to solve vessel collision risk assessment [7]. Experimental results show that the proposed approach confirms the validity and is reasonable for real application. But D-S evidence theory cannot solve the conflict evidence problem.

To solve this problem, according to the multiple experts judgment matrix by using analytic hierarchy process method and D-S evidence theory, avoid the conflict in information on the expert information synthesis from the actual case. According to the weight of the fusion, establish an expert weight target function and determine the weight of experts using genetic algorithms. Finally, determine the weight of final system by weighting. This algorithm is effective to 


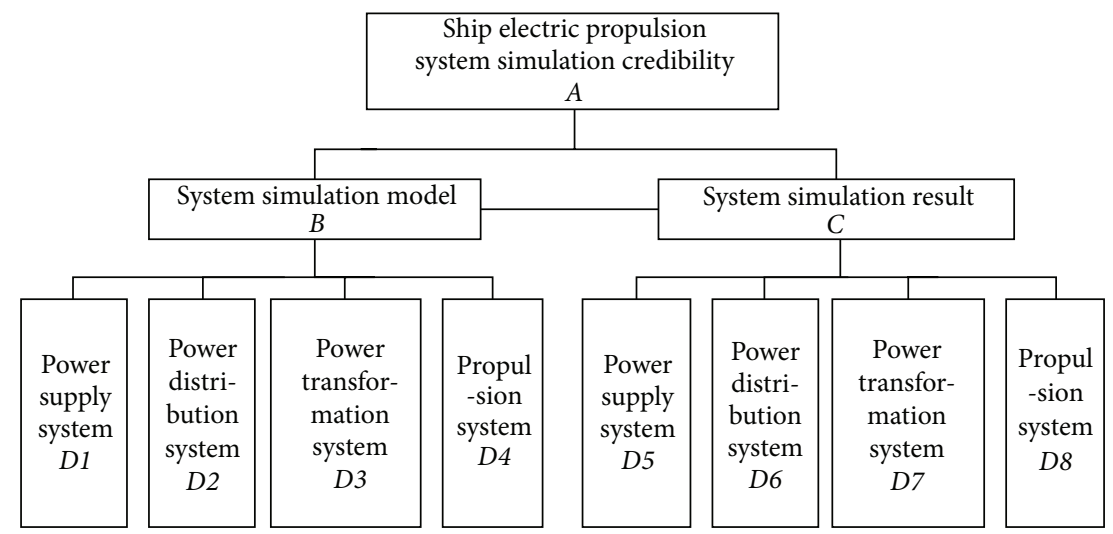

FIGURE 1: Structure diagram for ship electric propulsion simulation system reliability evaluation index system.

solve the synthesis expert information conflict problems and improve the system of the objectivity of the judgment.

\section{The Simulation System Reliability Evaluation Index System}

For the ship electric propulsion simulation system reliability evaluation study, according to user requirements and the characteristics of the system itself, first of all we need to establish the ship electric propulsion simulation system reliability evaluation index system. Based on the key indicators in research process, investigate subsystem one by one, and establish the system equivalence evaluation index system. In the process of building evaluation system, according to the target layer, criterion layer, and measures layer sequence, decompose the system step by step hierarchically and make a complicated problem decomposed into several elements. For ship electric propulsion simulation system is concerned about, target layer is ship electric propulsion simulation system reliability. Criterion layer, from the system simulation model to system based on the simulation results, determines the level of index measures step by step [8]. For system simulation model and system simulation results are concerned about, the reliability is analyzed from the power supply system, power distribution system, power transformation system, and propulsion system, respectively. Finally ship electric propulsion simulation system reliability evaluation system is shown in Figure 1.

\section{Expert Evaluation Weight Analysis}

\subsection{The Steps to Calculate the Index Weight}

Step 1. According to many experts' relative judgment matrix, use the principle of AHP to calculate the index of the initial weight.

Step 2. Determine the index weight fusion through the modified D-S fusion initial index weight, and determine the absolute judgment matrix through the expert judgment.
Step 3. According to each index fusion weight and absolute judgment matrix, determine the expert weight, the objective function and a genetic algorithm is adopted to calculate the optimal solution to determine the expert weight.

Step 4. Weigh the initial weight and expert weight, and determine the weights of the index.

3.2. Based on the AHP Analysis of Initial Weights. Initial weight can be calculated by AHP to carry out. Compared with the previous expert scoring method, fuzzy evaluation method, the grey correlation method, Pressure-StateResponse method (PSR), and artificial neural network algorithm, AHP is a kind of qualitative analysis and quantitative analysis and systematic and hierarchical multiple factors of decision analysis method; this method will be the decision maker's experience quantitative judgment. It is very convenient in the condition of the multiobjective and lack of necessary data [9].

Ship electric propulsion simulation system reliability evaluation index system calculation generally can be divided into the following four steps [10].

Step 1. Each element value in judgment matrix is relative to a certain element in a previous level, associated with the each elements in the layer pairwise comparison judgment importance. In the judge process, use 1-9 scale method to show, specific as is shown in Table 1.

Step 2. The element's relative weight for the criterion is calculated by judgment matrix.

Step 3. Compute synthetic weight of each of the layer elements to system target.

Step 4. Consistency check: consistency includes absolute consistency (or complete consistency) and order consistency. The so-called absolute consistency means that the judgment matrix $A$, If matrix A meet

$$
a_{i j}=a_{i k} a_{j k} \quad i, j, k=1,2, \ldots, n .
$$

We called matrix $A$ meet absolute consistency. 
TABLE 1: The scale method of 1 9.

\begin{tabular}{ll}
\hline Scale & Meaning \\
\hline 1 & $\begin{array}{l}\text { Two factors have the same importance } \\
\text { A factor relative to another factor a little } \\
\text { important }\end{array}$ \\
5 & $\begin{array}{l}\text { A factor relative to another obvious important } \\
\text { factors }\end{array}$ \\
7 & $\begin{array}{l}\text { A factor relative to another important factor } \\
\text { strongly }\end{array}$ \\
9 & $\begin{array}{l}\text { A factor relative to another extremely important } \\
\text { factor }\end{array}$ \\
$2,4,6,8$ & $\begin{array}{l}\text { Median in two adjacent judgments } \\
\text { Factors } i \text { and } j \text { are to judge } b_{i j} ; \\
\text { the factors } j \text { and } i \text { compare judgment } \\
b_{j i}=1 / b_{i j}\end{array}$ \\
\hline
\end{tabular}

It says $A$ is absolute consistency matrix (or complete consistency matrix); at the same time there is

$$
\begin{gathered}
a_{i j}=\frac{W_{i}}{W_{j}} \quad i, j, k=1,2, \ldots, n, \\
A W=n W .
\end{gathered}
$$

Sort consistency is to point to the following: if factor a is important than factor $b$ and factor $b$ is important than factor $c$, then a factor is important than factor $c$. And the consistency check index C.I. is as follows:

$$
\text { C.I. }=\frac{\lambda_{\max }-n}{n-1} .
$$

The $n$ is the order number of judgment matrix $A$ and $\lambda_{\max }$ is the biggest characteristic root of judgment matrix $A$.

Calculation consistency ratio C.R. is as follows:

$$
\text { C.R. }=\frac{\text { C.I. }}{\text { R.I. }}
$$

When C.R. < 0.1, consider the consistency of judgement matrix is acceptable [11] (Table 2).

\subsection{The Construction of Expert Weight Objective Function} Based on D-S Method. For the ship electric propulsion system concerned about, setting the index set $B=\left\{b_{1}, b_{2}, \ldots, b_{n}\right\}$, experts set $D=\left\{d_{1}, d_{2}, \ldots, d_{m}\right\}$, and matrix $A=\left(a_{i j}\right)_{m \times n}(0<$ $\left.a_{i j}<1\right)$ is absolute judgment matrix coming from experts to marking index weight. Set index fusion weight vector $W=$ $\left\{w_{1}, w_{2}, \ldots, w_{n}\right\}$, expert weight vector is $R=\left\{r_{1}, r_{2}, \ldots, r_{m}\right\}$, and meet $\sum_{j=1}^{n} w_{j}=1, \sum_{i=1}^{m} r_{i}=1$.

For absolute judgment matrix, if evaluation from expert $d_{i}$ has no difference with other experts' evaluation, the expert $d_{i}$ has a higher similarity with other experts. That is to say, expert $d_{i}$ has higher credibility. $F_{i j}(r)$ show the index judgment deviation from experts $d_{i}$ and other experts:

$$
F_{i j}(r)=\sum_{k=1}^{m}\left|a_{i j} r_{i}-a_{k j} r_{k}\right| * w_{j} .
$$

TABLE 2: The average random consistency targets R.I.

\begin{tabular}{lc}
\hline Matrix order number & R.I. \\
\hline 1 & 0 \\
2 & 0 \\
3 & 0.52 \\
4 & 0.89 \\
5 & 1.12 \\
6 & 1.26 \\
7 & 1.36 \\
8 & 1.41 \\
9 & 1.46 \\
10 & 1.19 \\
\hline
\end{tabular}

$F_{j}(r)$ show the total index judgment deviation from $m$ experts:

$$
F_{j}(r)=\sum_{i=1}^{m} \sum_{k=1}^{m}\left|a_{i j} r_{i}-a_{k j} r_{k}\right| * w_{j} \text {. }
$$

For index $b_{j}$, deviation value $F_{j}(r)$ is smaller; credibility of the judge from experts is higher. For the system in the index, total deviation value is smaller; credibility of the judge from experts is higher. According to the calculation of the system integration indicators weight, structural expert weight target optimization function

$$
\begin{array}{ll}
\min & F(r)=\sum_{j=1}^{n} \sum_{i=1}^{m} \sum_{k=1}^{m}\left|a_{i j} r_{i}-a_{k j} r_{k}\right| * w_{j} \\
\text { s.t. } \quad & \sum_{i=1}^{m} r_{i}=1 \\
& 0<r_{i}<1 \quad(i=1, \ldots, m) .
\end{array}
$$

3.4. Solve the Objective Function. Formula (7) belongs to nonlinear optimization problem, the genetic algorithm suitable for processing this kind of problem. With the increase of matrix dimension, parameters in formula (7) will increase sharply, in order to find the optimal solution under multivariate conditions, introducing the concept of niche to enhance the diversity of population $[12,13]$. Algorithm flow chart is shown in Figure 2.

\section{Ship Electric Propulsion Simulation System Expert Evaluation Weight Calculation Conclusion}

Based on the analysis of the weight of each subsystem, invite three authoritative experts to score evaluation. Relative to 


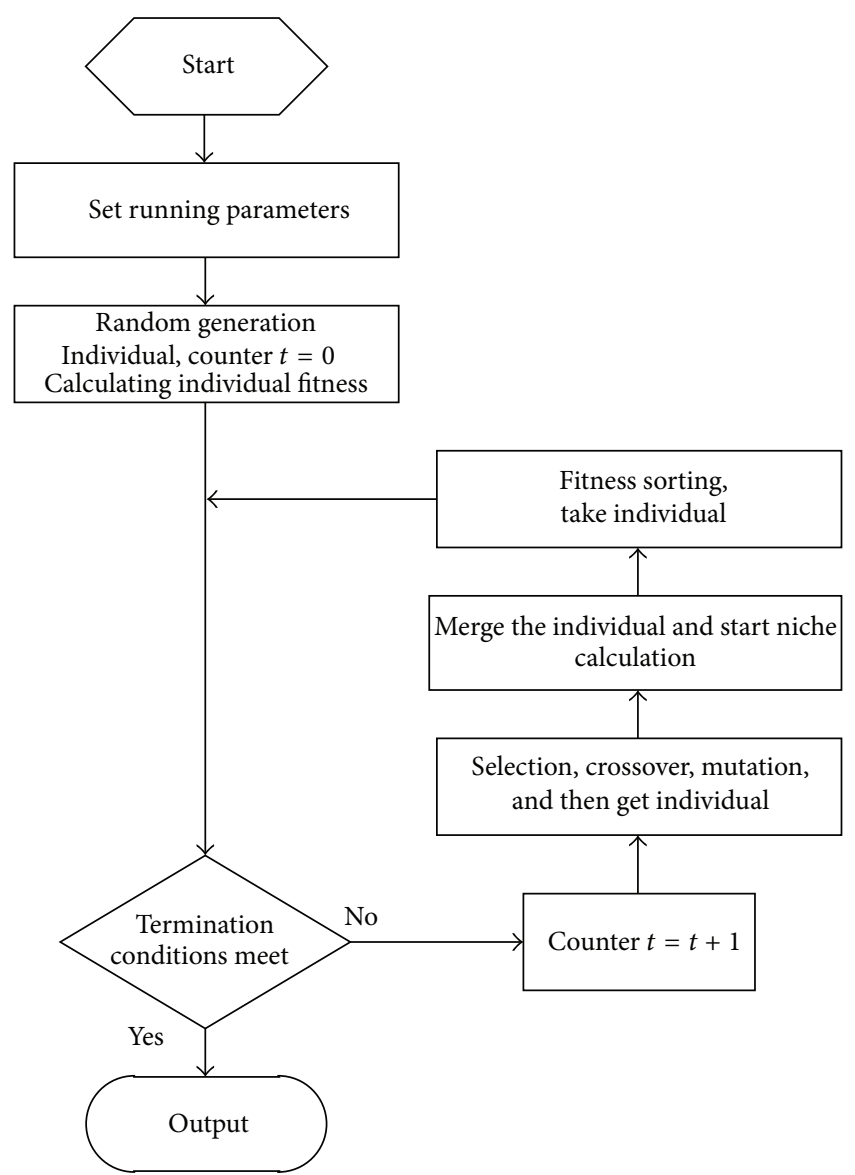

FIGURE 2: Flow chart for the niche genetic algorithm of AHP calculation.

the subsystem $B$, three experts are given, respectively, $D 1, D 2$, $D 3$, and D4 judgment matrix, specific as follows:

$$
\begin{aligned}
& B_{1}=\left[\begin{array}{llll}
1 & \frac{1}{6} & \frac{1}{3} & 3 \\
6 & 1 & 2 & 7 \\
3 & \frac{1}{2} & 1 & 5 \\
\frac{1}{3} & \frac{1}{7} & \frac{1}{5} & 1
\end{array}\right], \\
& B_{2}=\left[\begin{array}{llll}
1 & \frac{1}{2} & \frac{1}{2} & 3 \\
2 & 1 & 1 & 4 \\
2 & 1 & 1 & 4 \\
\frac{1}{3} & \frac{1}{4} & \frac{1}{4} & 1
\end{array}\right], \\
& B_{3}=\left[\begin{array}{llll}
1 & 1 & \frac{1}{5} & 3 \\
1 & 1 & \frac{1}{5} & 3 \\
5 & 1 & \frac{1}{7} & 7 \\
\frac{1}{3} & \frac{1}{3} & \frac{1}{7} & 1
\end{array}\right] .
\end{aligned}
$$

According to the principle of AHP calculate the corresponding initial weights of each subsystem, and check the consistency of judge matrix. If they do not meet the requirements for consistency, give the judge matrix again. Get three experts to $D 1, D 2, D 3$, and $D 4$ judgment weight: $W_{b 1}=\{0.1176,0.5345,0.2904,0.0578\}, W_{b 2}=\{0.2004,0.3587$, $0.3583,0.0816\}$, and $W_{b 3}=\{0.1953,0.1953,0.5324,0.0771\}$.

According to the calculation of the initial weight, using the improved D-S to fuse evaluation information, get $D 1, D 2$, $D 3$, and $D 4$ fusion weight to subsystem $B W_{b^{\prime}}=\{0.0633$, $0.3941,0.5334,0.0091\}$.

For ship electric propulsion system simulation model credibility analysis, the purpose is to determine whether the model is accurate. In this criterion, the three experts give $D 1, D 2, D 3$, and $D 4$ weight fuzzy evaluation matrix: $B=$

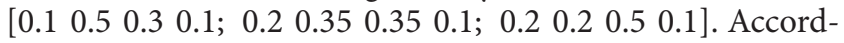
ing to each subsystem fusion weight and expert fuzzy evaluation matrix, by the algorithm to determine the expert weight objective function, and through the genetic algorithm optimization, determine the expert weight optimal solution. Get three subsystems D1, D2, D3, D4 weight evaluation of its own weight $R_{B}=\{0.401,0.36,0.238\}$.

According to the expert weight and the initial weight of the subsystem, using the weighted method to calculate the weight of each subsystem, get $D 1, D 2, D 3, D 4$ weight to subsystem $B W_{b}=\{0.1654,0.3886,0.3732,0.0706\}$.

Similarly, the three experts give the judgment matrix of each subsystem for objectives $A$ and $C$ and calculate the weight of each subsystem according to the above method. In view of the space reasons, give only the expert weight and the weight of each subsystem.

For the three experts grade evaluation for $D 5, D 6, D 7, D 8$, get the weight in the evaluation process of evaluation: $R_{C}=$ $\{0.48,0.218,0.3\}$, and the subsystem $D 5, D 6, D 7, D 8$ weight $W_{C}=\{0.1666,0.1758,0.2974,0.3582\}$.

Analyzing subsystem $B$ and $C$ against overall goal $A$, get evaluation weight $R_{A}=\{0.44,0.259,0.3\}$ and the weight subsystem $B, C$ to $A: W_{A}=\{0.5108,0.4882\}$.

According to the calculation of the $W_{A}, W_{B}, W_{C}$ weight vector, use the weighted method to calculate the total target:

$$
\begin{aligned}
& W=\left[\begin{array}{ll}
W_{B}^{\prime} & W_{C}^{\prime}
\end{array}\right] \cdot W_{A}^{\prime} \\
& =\left[\begin{array}{ll}
0.1653 & 0.1666 \\
0.3887 & 0.1758 \\
0.3731 & 0.2974 \\
0.0708 & 0.3582
\end{array}\right] \cdot\left[\begin{array}{l}
0.5108 \\
0.4882
\end{array}\right] \\
& =\left[\begin{array}{llll}
0.1658 & 0.2844 & 0.3358 & 0.2110
\end{array}\right]^{\prime} \text {. }
\end{aligned}
$$

From the calculation results above, it is known that, in the ship electric propulsion system, the simulation credibility is the greatest impacted by power conversion subsystem, secondly they were distribution subsystem, propulsion subsystem, and power subsystem. Power transformation simulation subsystem in energy conversion and harmonic aspects affect the credibility of the system. Distribution system simulation subsystem produces certain effect to management and distribution of electricity. Propulsion system simulation subsystem is aimed at mutation load. For power subsystem 
main consideration of its power quality, the other modules produce small amount of influence.

\section{Conclusions}

By using D-S theory and AHP, study the ship electric propulsion simulation system for the credibility evaluation expert weight. Through the inspection of the similarity between the experts, determine the expert weight objective function, and the genetic algorithm was used to calculate the expert weight optimal solution. This method not only can fuse the advantages of other methods, but also make better use of the expert advice in the evaluate process greatly. It can make subjective judgments of experts more united and avoid the one-sidedness when considering only one expert, and various judgments from different experts on reliability of ship electric propulsion simulation system can be treated; particularly evidence of conflict is no longer blindly negated. At the same time, this method can optimize the indexes and enhance the veracity and reliability for scientific decision-making, which has better comprehensive assessment evaluation and is more meaningful.

\section{Conflict of Interests}

The authors declare that there is no conflict of interests regarding the publication of this paper.

\section{Acknowledgments}

This work is supported by National Natural Science Foundation (NNSF) of China under Grant 51307026, Natural Science Foundation of Heilongjiang Province under Grant E201347, Fundamental Research Funds for the Central Universities under Grant HEUCFX41305, and the China Postdoctoral Funds (no. 2012M510924).

\section{References}

[1] L. Sheng and L. Lihong, "Application of FCE for credibility of simulation of ship electric propulsion system," Techniques of Automation \& Applications, vol. 32, no. 4, pp. 52-77, 2012.

[2] S. Ya-feng, W. Chao-yang, and H. Zhi-ping, "Research on simulation credibility," Electronic Measurement Technology, vol. 32, no. 11, pp. 8-11, 2009.

[3] H. Chen and W. Yong, "Credibility research of the simulation of the integrative avionics electronic system which based on cloud focus judgement," Electronic Measurement Technology, vol. 32, no. 10, pp. 69-72, 2009.

[4] B. Chen and J. Feng, "Multisensor information fusion of pulsed GTAW based on improved D-S evidence theory," International Journal of Advanced Manufacturing Technology, vol. 71, no. 1-4, pp. 91-99, 2014.

[5] Y. Chen, A. B. Cremers, and Z. Cao, "Interactive color image segmentation via iterative evidential labeling," Information Fusion, vol. 20, no. 1, pp. 292-304, 2014.

[6] L. Si, Z. Wang, C. Tan, and X. Liu, "A novel approach for coal seam terrain prediction through information fusion of improved D-S evidence theory and neural network," Measurement: Journal of the International Measurement Confederation, vol. 54, pp. 140-151, 2014.

[7] B. Li and F.-W. Pang, "An approach of vessel collision risk assessment based on the D-S evidence theory," Ocean Engineering, vol. 74, pp. 16-21, 2013.

[8] I.-S. Jung and C.-S. Lee, "Fuzzy inference and AHP-based alternative evaluation tool in the development of sustainable residential land," KSCE Journal of Civil Engineering, vol. 16, no. 3, pp. 273-282, 2012.

[9] C.-C. Sun, "A performance evaluation model by integrating fuzzy AHP and fuzzy TOPSIS methods," Expert Systems with Applications, vol. 37, no. 12, pp. 7745-7754, 2010.

[10] G.-Z. Li, N.-L. Tan, and J.-B. Zhang, "Criticality analysis of subway train equipment based on improved analytical hierarchy process," Journal of Electronic Measurement and Instrument, vol. 26, no. 6, pp. 503-507, 2012.

[11] W. Shi, Y. Li, C. Deng, M. Fan, and Z. Cai, "Design and implementation of water environment safety risk assessment model based on AHP," Chinese Journal of Scientific Instrument, vol. 30, no. 5, pp. 1009-1013, 2009.

[12] Z.-Y. Yang, J.-B. Qu, and C.-K. Huang, "Bridge safety evaluation based on fuzzy synthetic evaluation method and analytic hierarchy process," Journal of Tianjin University Science and Technology, vol. 38, no. 12, pp. 1063-1067, 2005.

[13] S. Liu, Y. Zhang, and D. Yu, "Correctinn consistency of fuzzy judgment matrix using niche genetic algorithm," Journal of Huazhong University of Science and Technology, vol. 38, no. 7, pp. 126-129, 2010. 


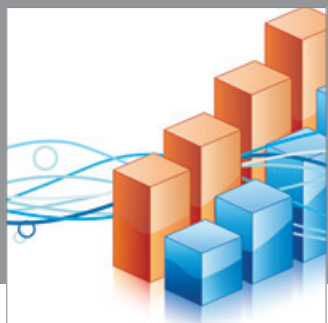

Advances in

Operations Research

mansans

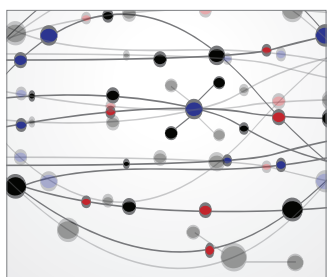

The Scientific World Journal
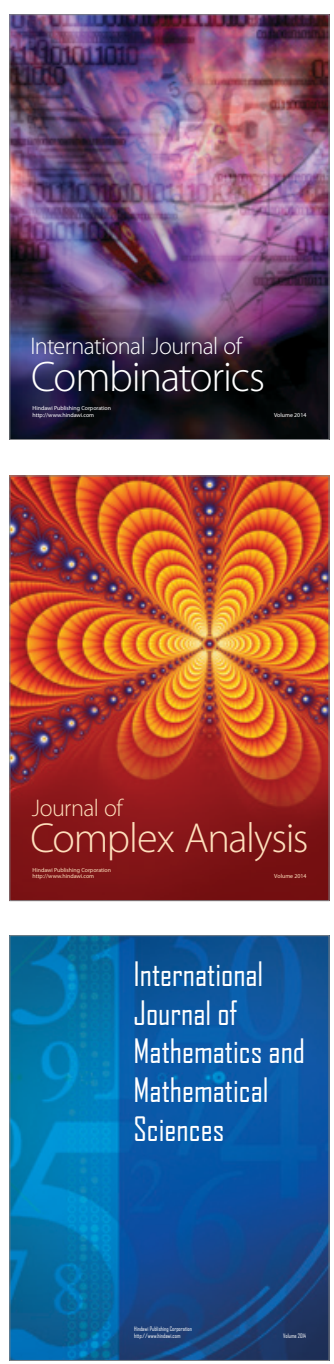
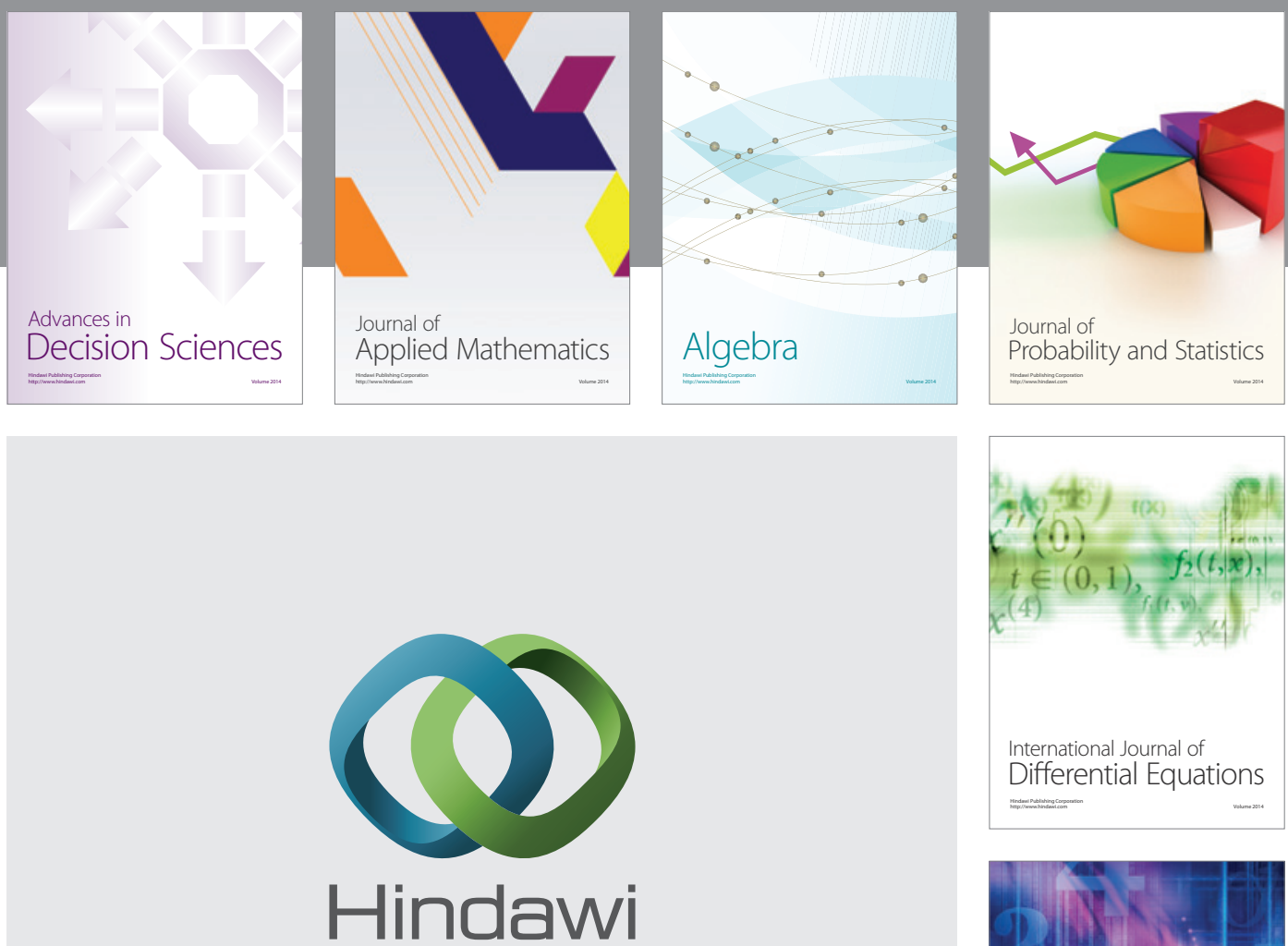

Submit your manuscripts at http://www.hindawi.com
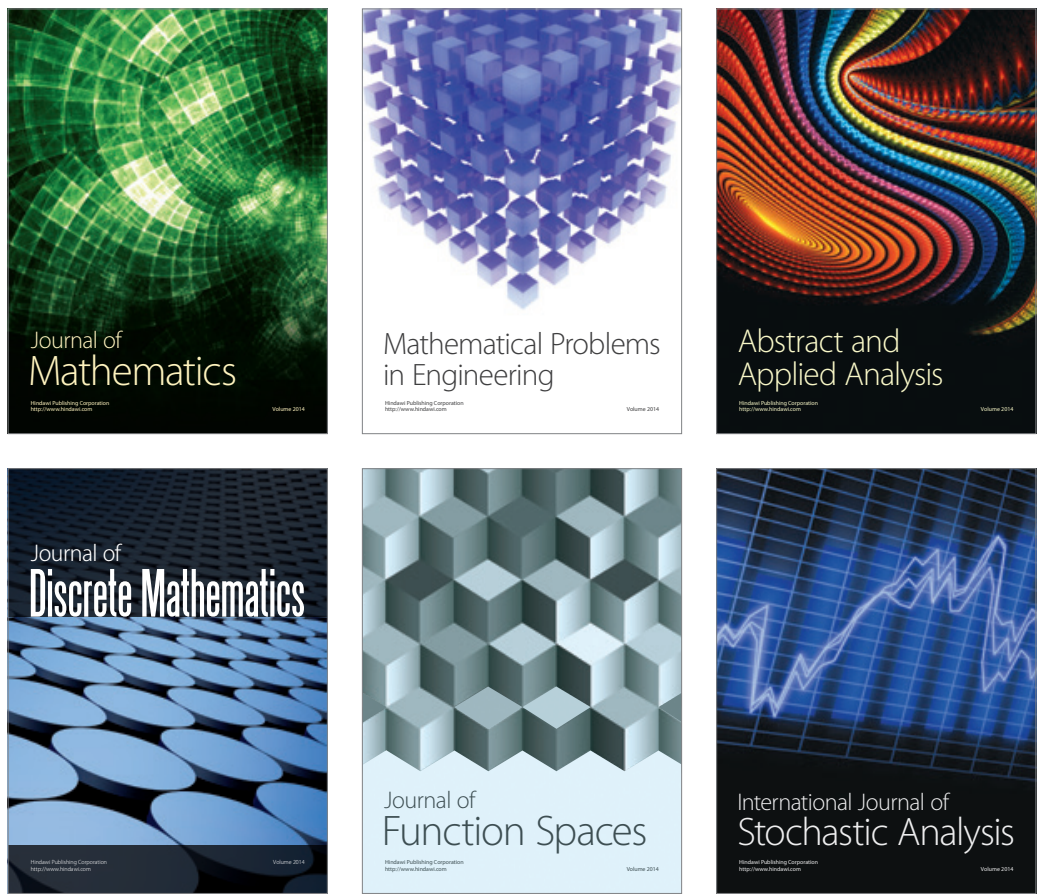

Journal of

Function Spaces

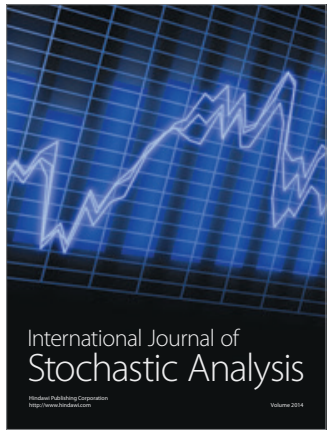

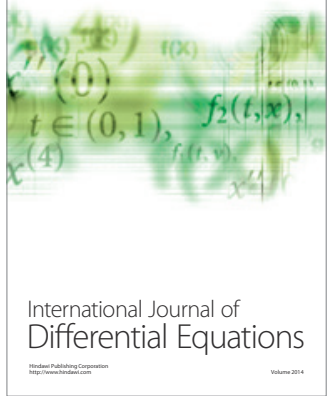
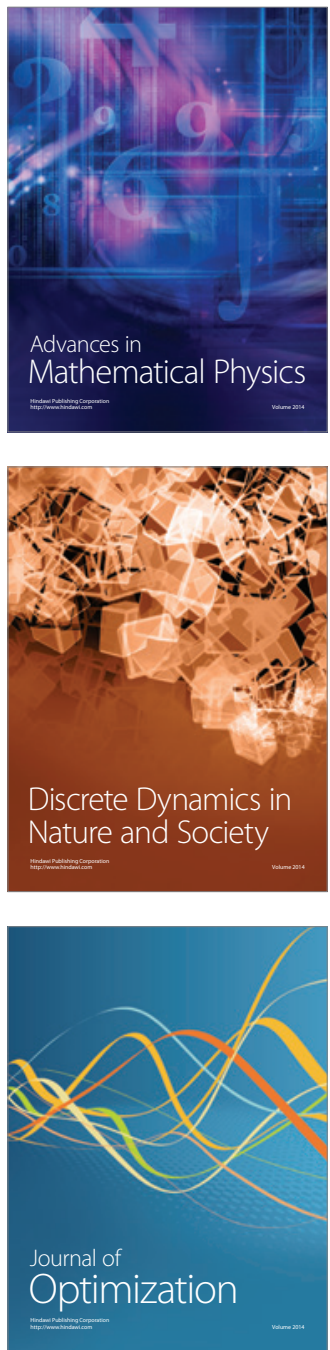